\title{
AN APPLICATION OF MÖBIUS INVERSION TO A PROBLEM IN TOPOLOGICAL DYNAMICS
}

\author{
BY NELSON G. MARKLEY AND WILLIAM N. ANDERSON, JR. \\ Communicated by Walter Gottschalk, March 5, 1973
}

1. Introduction. Let $X$ be a topological space and let $\phi$ be a homeomorphism of $X$ onto $X$. The pair $(X, \phi)$ is called a cascade. A nonempty subset $M$ of $X$ is a minimal subset of $(X, \phi)$ if $M$ is closed, $\phi(M)=M$, and no proper subset of $M$ has these properties. Equivalently $M(M \neq \varnothing)$ is minimal if and only if for every $x$ in $M$ we have $\mathrm{Cl}\left\{\phi^{n}(x): n \in Z\right\}=M$. A homomorphism of $(X, \phi)$ into $(Y, \psi)$ is a continuous map $\theta$ of $X$ into $Y$ such that $\theta \circ \phi=\psi \circ \theta$.

Let $K=\{z \in C:|z|=1\}$ and let $(K, \phi)$ be a cascade such that $\phi^{n}(x)=x$ implies $n=0$. Then $(K, \phi)$ has exactly one minimal set which is either all of $K$ or a Cantor subset $C$. We are only interested in the latter case, and we write

$$
C=K \backslash \bigcup_{n=1}^{\infty}\left(a_{n}, b_{n}\right)
$$

when $\left(a_{n}, b_{n}\right)$ are counterclockwise open intervals in $K$ and

$$
\left[a_{n}, b_{n}\right] \cap\left[a_{m}, b_{m}\right]=\varnothing
$$

whenever $n \neq m$. Note that $\phi\left[\left(a_{i}, b_{i}\right)\right]=\left(\phi\left(a_{i}\right), \phi\left(b_{i}\right)\right)=\left(a_{j}, b_{j}\right)$ for some $j \neq i$. Thus $\phi$ defines an equivalence relation on the complementary intervals $\left\{\left(a_{n}, b_{n}\right): n=1, \ldots\right\}$. The restriction of $\phi$ to $C$ is a homeomorphism of $C$ onto $C$ and produces a minimal cascade which we denote by $(C, \phi)$.

A cascade $(X, \psi)$ on a compact Hausdorff space will be called an $n$-extension of $(C, \phi)$ if there exists an open $n$-to-one homomorphism of $(X, \psi)$ onto $(C, \phi)$. If the number of equivalence classes of complementary intervals of $C$ is finite, then for each positive integer $n$ the number of isomorphism classes of minimal $n$-extensions is finite [3, Corollary 6.6]. Let $\mathscr{I}(C, \phi, n)$ denote this number. We consider the problem of determining $\mathscr{I}(C, \phi, n)$ or an asymptotic expression for it as $n$ goes to infinity.

In the next section we present some combinatorial results which we applied to this problem, and in the last section we present our results on $\mathscr{I}(C, \phi, n)$. Proofs and tables of values can be found in [1].

2. Combinatorial results. Let $N$ be a set with $n$ elements, and let $\mathscr{S}_{n}$ be the symmetric group of all permutations acting on $N$. Let $\mathscr{S}_{n}^{k}$ be the set

AMS (MOS) subject classifications (1970). Primary 54H20, 05 A15. 
of all $k$-tuples of members of $\mathscr{S}_{n}$, and let $T$ be the subset of $\mathscr{S}_{n}^{k}$ consisting of all $v=\left(v_{1}, \ldots, v_{k}\right)$ such that:

(1) $v_{i} \neq v_{i+1}$ and $v_{k} \neq v_{1}$

(2) the components of $v$ generate a transitive group.

Finally, let $\mathscr{S}_{n}$ act on $T$ by conjugation. The number $M(n, k)$ is defined to be the number of orbits determined in $T$ by this action.

Using Burnside's theorem [2] we obtain the following inequality:

$$
\frac{\theta_{k}(n !)}{n !}-\sum_{j=1}^{[n / 2]} \frac{\theta_{k}(i !(n-i) !)}{i !(n-i) !} \leqq M(n, k) \leqq \sum_{d \mid n} \frac{\theta_{k}\left[(n / d)^{d} d !\right]}{(n / d)^{d} d !}
$$

where $\theta_{k}$ is the chromatic polynomial $\theta_{k}(s)=(s-1)^{k}+(-1)^{k}(s-1)$. From this we obtain

$$
\lim _{k \rightarrow \infty} \frac{n ! M(n, k)}{(n !-1)^{k}}=1, \quad \lim _{n \rightarrow \infty} \frac{M(n, k)}{(n !)^{k-1}}=1 .
$$

The cardinality $|T|$ of $T$ is given by

$$
n ! \sum_{p(n)} \frac{(-1)^{\alpha_{1}+\cdots+\alpha_{m}-1}\left(\alpha_{1}+\cdots+\alpha_{m}-1\right) !}{1 !^{\alpha_{1}} \cdots m !^{\alpha_{m}} \alpha_{1} ! \cdots \alpha_{m} !} \theta_{k}\left(1 !^{\alpha_{1}} \cdots m !^{\alpha_{m}}\right)
$$

where the sum is extended over all partitions of the integer $n$. This formula is derived by using Möbius inversion on the lattice of all partitions of the set $N$. The necessary Möbius function has been computed by Rota [4]. When $n$ is prime we obtain the following exact formula for $M(n, k)$ :

$$
M(n, k)=|T|+\sum_{d \mid n} \mu(d) \theta_{k}(n / d),
$$

where $\mu$ is the classical Möbius function.

Let $T_{0}$ be the subset of $T$ such that $v_{k}=v_{1}^{-1} v_{2} v_{1}$, let $\mathscr{S}_{n}$ act on $T_{0}$ by conjugation, and let $E(n, k)$ denote the number of orbits determined in $T_{0}$ by this action. The computation of $E(n, k)$ is similar to that for $M(n, k)$ except that a different chromatic polynomial is involved.

3. Dynamical results. The assumption that the number of equivalence classes of complementary intervals of $C$ determined by the action of $\phi$ is finite will always be in force. This number will be denoted by $m$.

Let $\mathscr{C}(C, \phi, n)$ denote the number of minimal cohomology classes of $(C, \phi)$ for $\mathscr{S}_{n}$. Using the results in [3] one can pick canonical representatives for the minimal cohomology class of $(C, \phi)$ for $\mathscr{S}_{n}$ in such a way that they can be counted using the ideas in the previous section. First we obtain the inequality

$$
M(n, m+1) \leqq \mathscr{C}(C, \phi, n) \leqq 1+\sum_{k=2}^{m+1}\left(\begin{array}{c}
m+1 \\
k
\end{array}\right) M(n, k)
$$


and from this we obtain $\lim _{n \rightarrow \infty} \mathscr{C}(C, \phi, n) /(n !)^{m}=1$.

THEOREM 1. If the only automorphisms of $(C, \phi)$ are of the form $\phi^{k}$, then $\mathscr{I}(C, \phi, n)=\mathscr{C}(C, \phi, n)$ and $\lim _{n \rightarrow \infty} \mathscr{I}(C, \phi, n) /(n !)^{m}=1$.

When $m=1$ the minimal set $(C, \phi)$ is a Sturmian minimal set. In this case $\mathscr{I}(C, \phi, n)=1+M(n, 2)$ and when $n$ is $2,3,4,5,6,7$ then $\mathscr{I}(C, \phi, n)$ is $3,7,26,97,624,4157$.

Finally, we obtain an exact formula.

THEOREM 2. If all the automorphisms of $(C, \phi)$ are of the form $\phi^{k}$, then

$$
\begin{aligned}
\mathscr{I}(C, \phi, n)=1 & +\left(\begin{array}{c}
m+1 \\
2
\end{array}\right) M(n, 2)+\sum_{k=3}^{m}\left(\begin{array}{l}
m \\
k
\end{array}\right) M(n, k) \\
& +\sum_{k=2}^{m}\left(\begin{array}{l}
m \\
k
\end{array}\right)[M(n, k+1)-E(n, k+1)] .
\end{aligned}
$$

\section{REFERENCES}

1. W. N. Anderson and N. G. Markley, An application of Mobius inversion to a problem in topological dynamics, University of Maryland, Department of Mathematics, Technical Report TR 72-12.

2. C. L. Liu, Introduction to combinatorial mathematics, McGraw-Hill, New York, 1968. MR 38 \#3154.

3. N. G. Markley, Locally circular minimal sets, Pacific J. Math. 46 (to appear).

4. G.-C. Rota, On the foundations of combinatorial theory. I. Theory of Möbius functions, Z. Wahrscheinlichkeitstheorie und Verw. Gebiete 2 (1964), 340-368. MR 30 \#4688.

Department of Mathematics, University of Maryland, College Park, Maryland 20742 